\title{
The threshold-photoelectron spectrum of $\mathrm{NH}_{3}$
}

\author{
R. Locht \\ Département de Chimie Générale et de Chimie Physique, Institut de Chimie, Bâtiment B6, Université de Liège, \\ Sart-Tilman par, 4000 Liege 1, Belgium \\ K. Hottmann, G. Hagenow, W. Denzer and H. Baumgartel \\ Institut fiir Physikalische und Theoretische Chemie, Freie Universität Berlin, Takustrasse 3, W-1000 Berlin 33, \\ Germany
}

\begin{abstract}
Using synchrotron radiation, we have investigated the threshold-photoelectron spectrum of $\mathrm{NH}_{3}$ between 10 and $30 \mathrm{eV}$ photon energy. In contrast to the $\mathrm{He}(\mathrm{I})$-photoelectron spectrum, the threshold-photoelectron spectrum shows three bands between 10 and $21 \mathrm{eV}$ photon energy. The first band is dominated by the vibrational autoionization of the $5 \mathrm{sa}_{1}$, the $6 \mathrm{sa}_{1}$ and the 6 pe Rydberg series. Between the first and second band, a new contribution, starting at $13.26 \mathrm{eV}$, is assigned to the autoionization of (a) member(s) of Rydberg series converging to the $\mathrm{e}^{-1}$ ionization continuum. Its (their) autoionization populates the upper vibrational levels of the $\mathrm{NH}_{3}{ }^{+}\left(\tilde{X}^{2} \mathrm{~A}_{1}\right)$ state. From about $14.5 \mathrm{eV}$ the $\tilde{\mathrm{A}}^{2} \mathrm{E}$ band structures are identified. However, differences between the $\tilde{\mathrm{A}}^{2} \mathrm{E}$ band shapes in the $\mathrm{He}(\mathrm{I})$ - and the threshold-photoelectron spectra suggest that very likely autoionization contributions are involved in the latter spectrum. These phenomena could play a role in the production of the $\mathrm{NH}_{2}{ }^{+}$and $\mathrm{NH}^{+}$fragment ions. The last very weak band, at about $25 \mathrm{eV}$ in the photoelectron spectrum, is assigned to the $\mathrm{NH}_{3}^{+}\left(\tilde{\mathrm{B}}^{2} \mathrm{~A}_{1}\right)$ state.
\end{abstract}

\section{Introduction}

In the framework of our study devoted to the dissociative ionization of $\mathrm{NH}_{3}$ [1-3], the photoionization efficiency and the translational energy of $\mathrm{NH}_{2}^{+}$and $\mathrm{NH}^{+}$fragment ions were measured. Weak structures were observed in the photoion yield curve $\mathrm{NH}_{2}{ }^{+}$above the onset energy at $15.75 \mathrm{eV}$ [4]. Furthermore, in the energy range of the $\mathrm{NH}_{2}{ }^{+}$onset energy, the photoionization efficiency curve of $\mathrm{NH}_{3}{ }^{+}$shows several weak structures [4].

To pursue our effort to understand these phenomena, the autoionization in $\mathrm{NH}_{3}$ has been investigated in detail. For this purpose, first the photoionization efficiency curves of $\mathrm{NH}_{3}$ and its three isotopomers have been measured in the energy range of the first ionic $\tilde{X}^{2} \mathrm{~A}_{1}$ state [5]. The abundant autoionization structure has been analyzed in terms of Rydberg series converging to the first ionization limit. These superexcited states, characterized by extensive vibrational progressions, autoionize vibrationally. To interprete the autoionization spectrum, it was assumed that autoionization occurs through transitions to the nearest ionization continuum, giving rise to low-energy electrons.

To test this assumption and to investigate the autoionization at higher energies, essentially in the vicinity of the appearance energies of $\mathrm{NH}_{2}^{+}$at $15.7 \mathrm{eV}$ [1,4], and of $\mathrm{NH}^{+}$at $16.9 \mathrm{eV}$ [1,4], the threshold photoelectron spectrum of $\mathrm{NH}_{3}$ has been measured. For comparison, a high-resolution $\mathrm{He}(\mathrm{I})$-photoelectron spectrum has also been recorded.

\section{Experimental}

The experimental setup and the experimental conditions used in this work have been outlined earlier [6] and will be described in detail in a forthcoming paper [7]. Only the most salient features will be laid out here.

Both the $\mathrm{He}(\mathrm{I})$ - and the threshold-photoelectron spectra were measured by means of the same highresolution tandem electron spectrometer made of two $180^{\circ}$ electrostatic deflectors. The dispersion being 104 , a theoretical resolution $\Delta E / E=0.5 \%$ is obtained with $0.5 \mathrm{~mm}$ slitwidths.

For the threshold-photoelectron spectra, recorded at a pass energy of $10 \mathrm{~V}$, the resolution (at fwhm) is 25-30 meV as measured on the asymmetric zero-kinetic energy $\operatorname{Ar}^{+}\left({ }^{2} \mathrm{P}_{1 / 2}\right)$ peak. This signal is used for the wavelength calibration of a 3 meter normal-incidence monochromator ( $3 \mathrm{~m}$ NIM-1), equipped with a platinum 
grating of $1200 \mathrm{l} / \mathrm{mm}$, and used to disperse the synchrotron radiation provided by the electron storage ring BESSY (Berlin). Owing to the low transmission of the Pt-grating, particularly in the 100-120 nm wavelength region, the entrance and the exit slits were set at 200 and $600 \mu \mathrm{m}$, respectively. The threshold-photoelectron spectra were normalized to the light intensity measured by a sodium salycilate-sensitized photomultiplier.

For the $\mathrm{He}(\mathrm{I})$-photoelectron spectrum, measured at a band pass energy of $1.9 \mathrm{~V}$, a resolution of $15 \mathrm{meV}$ is obtained (at fwhm of the $\mathrm{Xe}^{2} \mathrm{P}_{3 / 2}$ ) peak. A microwave discharge lamp is used as a light source. The energy calibration is the result of a linear regression on the four peaks observed in the spectrum of a $\mathrm{NH}_{3}, \mathrm{Xe}$ and $\mathrm{Kr}$ mixture. The accuracy is about $2 \mathrm{meV}$ for the first band and somewhat less for the second band.

The ammonia sample used in this work was of commercial quality (Linde A.G.) of $99.95 \%$ purity and introduced without further purification. The sample pressure in the ionization region was about $5 \times 10^{-6} \mathrm{mbar}$.

Fig. 1. Threshold-photoelectron spectrum of $\mathrm{NH}_{3}$ in the $10-30 \mathrm{eV}$ photon energy range. $\tilde{\mathbf{X}}^{2} A_{l}, \tilde{A}^{2} E$ and $\tilde{\mathrm{H}}^{2} A_{1}$ refer to the energy ranges of the corresponding ionic states in the He(I)-photoelec-tron spectrum.

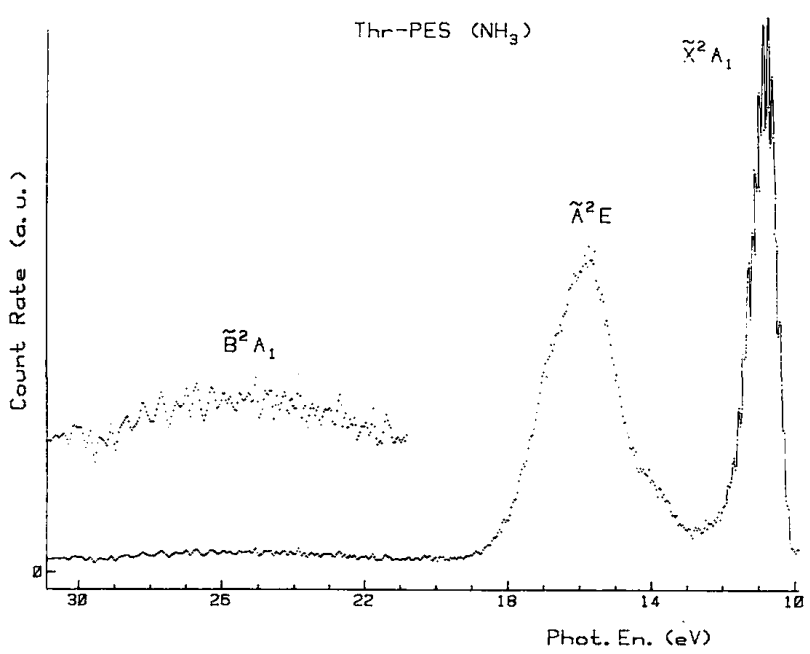

Fig. 2. Threshold-photoelectron spectrum of $\mathrm{NH}_{3}$ in the 10-12 eV photon-energy range. Vertical bars locate the vibrational progressions of the He(I) potential energy surface (PES) and the $5 \mathrm{~s} a_{1}$, 6sa $a_{1}$, 6pe and 7pe Rydberg states.

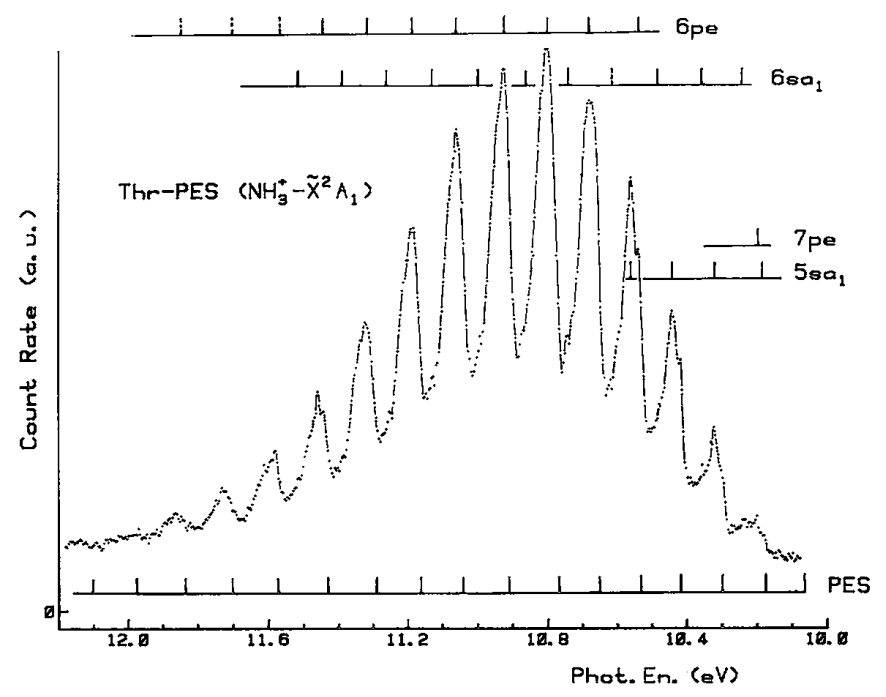


Table 1. Energy (eV) and assignment of the structure observed in the first threshold-photoelectron band of $\mathrm{NH}_{3}$. Comparison is made with the mass spectrometric photoionization [ 5 ] and He(I)-photoelectron spectroscopic results. The symbol $\tilde{\mathrm{X}}_{\mathrm{X}}$ refers to the $\tilde{\mathrm{X}}^{2} \mathrm{~A}_{1}$ state of $\mathrm{NH}_{3}{ }^{+}$. The error in the data of the present PES-work is \pm 0.002 $\mathrm{eV}$ and of the TPES- and Pi-work is $\pm 0.010 \mathrm{eV}$. (M) refers to the maxima of the peaks

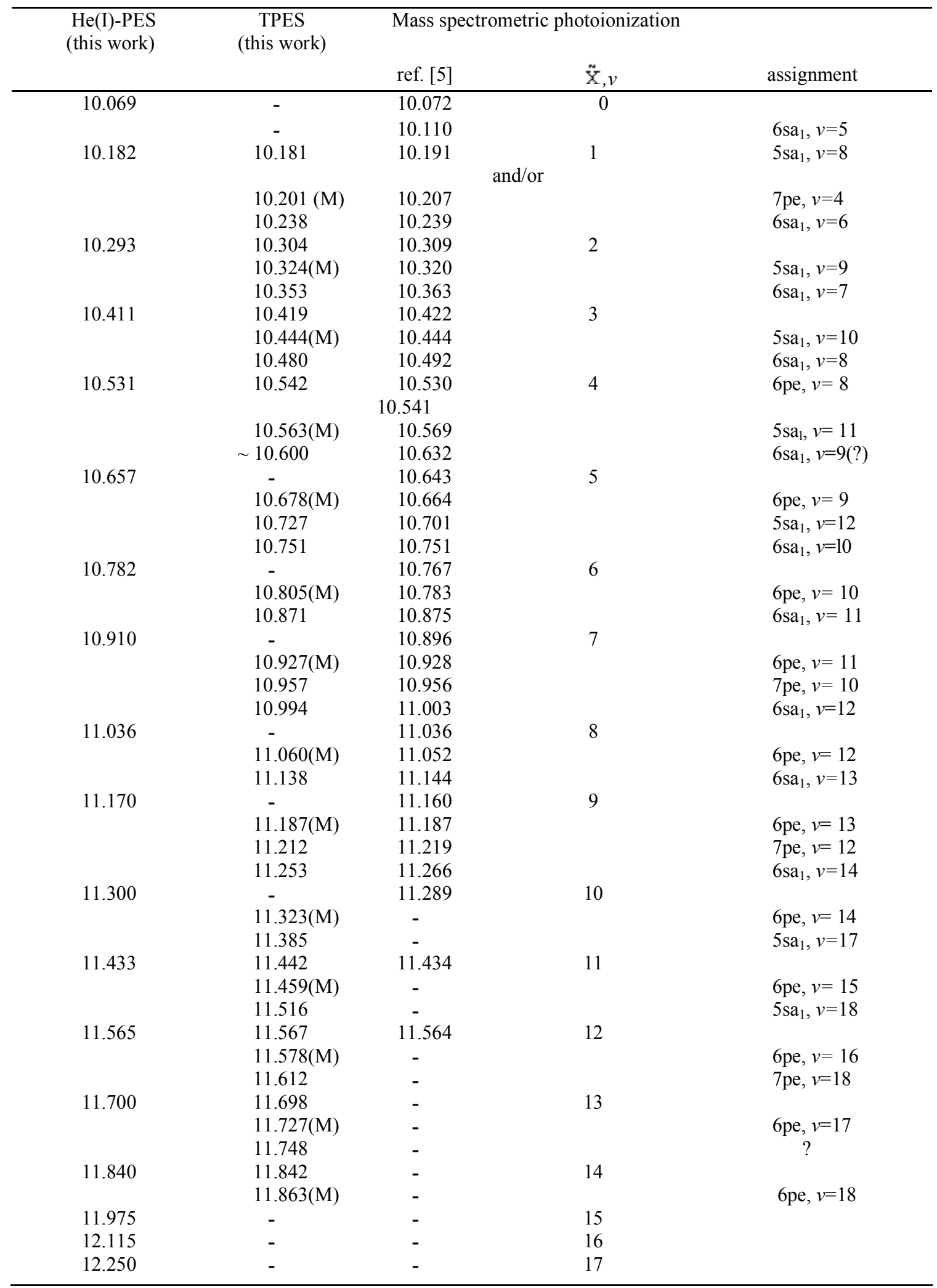




\section{Discussion}

The threshold-photoelectron spectrum of $\mathrm{NH}_{3}$, in the 10 to $30 \mathrm{eV}$ photon energy range, is displayed in fig. 1. This spectrum exhibits three bands covering roughly the energy ranges of the $\ddot{\mathrm{X}}^{2} \mathrm{~A}_{1}$, the $\tilde{\mathrm{A}}^{2} \mathrm{E}$ and the $\ddot{\mathrm{B}}^{2} \mathrm{~A}_{1}$ states of $\mathrm{NH}_{3}{ }^{+}$at about 11, 16 and $25 \mathrm{eV}$, respectively. However, on the low-energy side of the second band, and starting at $13 \mathrm{eV}$, a fairly intense shoulder is observed in the threshold-photoelectron spectrum. This feature is absent in the well-known $\mathrm{He}(\mathrm{I})$-photoelectron spectrum of $\mathrm{NH}_{3}$ [8] where the $\tilde{\mathrm{A}}^{2} \mathrm{E}$ state is characterized by an adiabatic ionization energy of $14.725 \mathrm{eV}[8]$.

The first threshold-photoelectron band has been recorded on a more expanded energy scale for a detailed analysis (see fig. 2).

Owing to the unfavourable transmission of the Pt-grating in the wavelength region of 100-120 nm, very long measuring times were required to obtain an acceptable signal-to-noise ratio. The general band shape looks quite similar to the $\mathrm{He}(\mathrm{I})$-photoelectron spectrum of the $\tilde{\mathrm{x}}^{2} \mathrm{~A}_{1}$ state. However, each partially resolved vibrational band, separated by 120-140 meV intervals, clearly shows fine structures. On the other hand, the photoelectron spectrum recorded at fixed wavelength $(65 \mathrm{~nm}$ or $19.929 \mathrm{eV})$ and under the same experimental conditions showed a quasi fully resolved smoothly shaped vibrational structure at 110-120 meV intervals.

The adiabatic ionization transition is not observed in the present work, probably on account of the weakness of the signal, whereas it is observed by photoionization [5], in the $\mathrm{He}(\mathrm{I})$-photoelectron spectrum and in the $65 \mathrm{~nm}$ photoelectron spectrum recorded during this work. Above the $0 \leftarrow 0$ transition at $10.072 \mathrm{eV}$ [5] fourteen structured peaks are detected and the position in energy of these features is listed in table 1 together with the results obtained for the $\mathrm{He}(\mathrm{I})$-photoelectron spectrum.

The first four peaks show a steeply rising shoulder or a slightly resolved peak on the low-energy side. The fairly good agreement between the present measurements and the earlier data [5] allows us to ascribe these features to the production of zero-kinetic energy electrons through the direct ionization of $\mathrm{NH}_{3}$ to $\mathrm{NH}_{3}^{+}\left(\tilde{\mathrm{X}}^{2} \mathrm{~A}_{1}, v=1\right.$ to 4 ). However, at $10.181 \mathrm{eV}$, a contribution of the $5 \mathrm{sa}_{1}, v=8 \leftarrow 3 \mathrm{a}_{1}$ cannot be excluded. At $10.542 \mathrm{eV}$, the assignment is uncertain: both $\mathrm{NH}_{3}{ }^{+}\left(\overrightarrow{\mathrm{X}}^{2} \mathrm{~A}_{1}, v=4\right)$, measured at $10.531 \mathrm{eV}$ by photoelectron spectroscopy, and the autoionization of the (6pe, $v=8)$, measured at $10.541 \mathrm{eV}$ [5], could be involved. The remaining structure has to be assigned to autoionization electrons from mainly the $5 \mathrm{sa}_{1}$ and $6 \mathrm{sa}_{1}$ Rydberg states. At $10.201 \mathrm{eV}$, the $7 \mathrm{pe}, v=4$ is probably involved.

Up from the fifth peak, broadened and irregularly shaped peaks are observed. Weak shoulders are detected on the high-energy side. For the interpretation and assignment of these features, their energies have to be compared to the photoionization results [5] listed in table 1. In most cases, the good agreement between the present data and the mass spectrometric photoionization measurements indicates autoionization contributions from mainly the $5 \mathrm{sa}_{1}(v)$, the $6 \mathrm{sa}_{1}(v)$ and $6 \mathrm{pe}(v)$ Rydberg states. The position of the maxima of the peaks agrees with the 6pe vibrational progression from $v=9$ to $v=13$. The zero-kinetic-energy electrons corresponding to $\mathrm{NH}_{3}{ }^{+}$ $\left(\tilde{\mathrm{X}}^{2} \mathrm{~A}_{\mathrm{l}}, v=5\right.$ to 10$)$ are not resolved as clearly as at lower energies. These are probably buried in the signal of the autoionization electrons.

Above $v=10$ in table 1 , a number of structures are identified in the threshold-photoelectron spectrum, whereas the autoionization observed in the photoionization work could only be analyzed up to $11.2 \mathrm{eV}$ photon energy [5]. However, to interprète and assign the features above $11.3 \mathrm{eV}$, data obtained from photoionization will be used, i.e. the term values $T$ characterizing the $5 \mathrm{sa}_{1}(T=0.859 \mathrm{eV}), 6 \mathrm{sa}_{1}(T=0.548 \mathrm{eV}), 6 \mathrm{pe}(T=0.511 \mathrm{eV})$ and 7 pe $(T=0.353 \mathrm{eV})$ Rydberg series [5].

As shown in table 1 up from $v=11$, besides the direct ionization to $\mathrm{NH}_{3}^{+}\left(\tilde{x}^{2} \mathrm{~A}_{1}, v=11\right.$ to 14), which is again resolved, the maxima of the peaks are well fitted by the 6 pe vibrational progression up to $v=18$. Less accurately, the remaining structure is assigned to $5 \mathrm{sa}_{1}(v=17$ and 18$)$ and $7 \mathrm{pe}(v=18)$.

The second threshold-photoelectron band, represented on an expanded energy scale in fig. 3, starts at $13.26 \mathrm{eV}$ and extends to $19 \mathrm{eV}$. As mentioned earlier in this paper the most important difference between the present result and the $\mathrm{NH}_{3}{ }^{+}\left(\tilde{\mathrm{A}}^{2} \mathrm{E}\right)$-photoelectron band [8] is the broad and quasi-structureless shoulder on the low-energy side of the band starting at $13.26 \mathrm{eV}$. The $\mathrm{NH}_{3}{ }^{+}\left(\tilde{\mathrm{A}}^{2} \mathrm{E}\right)$ has an adiabatic ionization energy of 14.725 
[8] or $14.776 \mathrm{eV}$ as measured in this work (marked by a vertical line in fig. 3). Noteworthy is also the difference in band shape. The $\mathrm{He}(\mathrm{I})$-photoelectron spectrum of the $\tilde{\mathrm{A}}^{2} \mathrm{E}$ band shows a low-intensity structure superposed on a fairly flat plateau between 16 and $17.1 \mathrm{eV}$. In the threshold-photoelectron spectrum, the second band peaks at $16.2 \mathrm{eV}$ and decreases continuously to $19 \mathrm{eV}$.

Fig. 3. Threshold-photoelectron spectrum of $\mathrm{NH}_{3}$ in the 12-18 eV photon-energy range. Dashed lines indicate presumed fine structure. Full lines correspond to the energy position of the structures in the $\tilde{A}^{2} E \mathrm{He}(I)-P E$ band.

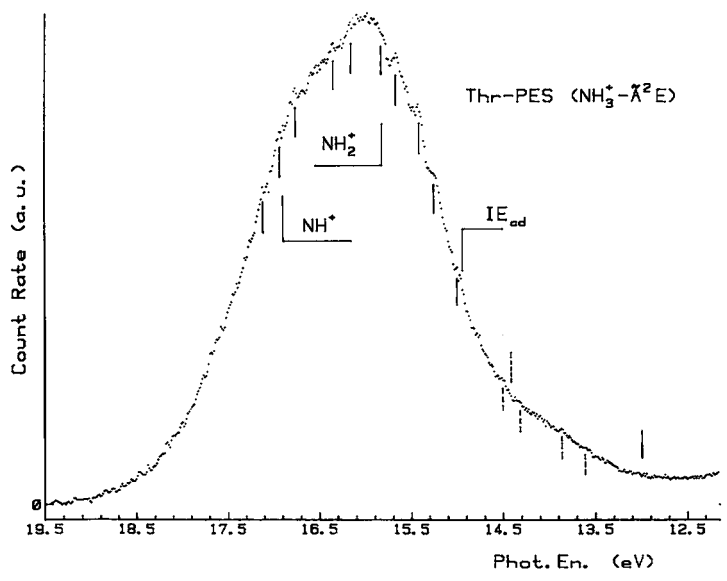

The shoulder observed between 13.26 and $14.6 \mathrm{eV}$ could be interpreted in two different ways: (i) the adiabatic ionization of the $\mathrm{NH}_{3}^{+}\left(\tilde{\mathrm{A}}^{2} \mathrm{E}\right)$ state is not reached in the Franck-Condon transition as observed by $\mathrm{He}(\mathrm{I})$ photoelectron spectroscopy or (ii) through autoionization, the $\mathrm{NH}_{3}^{+}\left(\mathrm{X}^{2} \mathrm{~A}_{1}\right)$ state is populated in the upper region of its potential hypersurface, outside the Franck-Condon region. The shoulder or slope change at about $14.6 \mathrm{eV}$ would argue for the latter interpretation. A more regular band shape would be expected in the case of the former hypothesis. The same kind of observations has already been made in the threshold-photoelectron spectra of $\mathrm{N}_{2} \mathrm{O}$ [9] and $\mathrm{C}_{2} \mathrm{H}_{2}[10]$.

On the other hand, in both cases the new contribution should be ascribed to autoionization of $\mathrm{NH}_{3}$ * superexcited state(s). This (these) state(s), probably of Rydberg character, should be (a) member(s) of series converging to the $\mathrm{e}^{-1}$ ionization continuum, i.e. $14.725 \mathrm{eV} \mathrm{[8]} \mathrm{or} 14.776 \mathrm{eV}$ was measured in this work. Using the onset at $13.26 \mathrm{eV}$ and the adiabatic ionization energy of the $\tilde{\mathrm{A}}^{2} \mathrm{E}$ state, we obtain a term value $T=1.465 \mathrm{eV}$, which is compatible with the term value for a 4 s Rydberg orbital, i.e. $T=1.532 \mathrm{eV}$ using $\delta=1.02$ [5].

The existence of superexcited state(s) in this energy range could find strong support by the observation of a fairly intense and broad band between 13.3 and $14.5 \mathrm{eV}$ in the electron energy-loss spectrum (EELS) of $\mathrm{NH}_{3}$ obtained by Furlan [11]. This up-to-now unassigned band is followed by a second broad band of about the same intensity, peaking at $15 \mathrm{eV}$ and decreasing smoothly to $18 \mathrm{eV}$ electron energy. No fine structure is observed in both features, though the electron energy resolution obtained in that work was about $25 \mathrm{meV}$ [11]. If a few discrete structures, i.e. peak and step-like features (see dashed lines in fig. 3) are recognized, the additional band observed in the threshold photoelectron spectrum looks essentially structureless at the present level of resolution.

The observation of the band spreading from 14.5 to $18 \mathrm{eV}$ in the electron energy-loss spectrum could suggest its contribution to the threshold-photoelectron spectrum through autoionization to the $\mathrm{NH}_{3}^{+}\left(\tilde{\mathrm{A}}^{2} \mathrm{E}\right)$ state. This phenomenon could be responsible for the particular shape of the $\tilde{\mathrm{A}}^{2} \mathrm{E}$ band in the threshold-photoelectron spectrum. Indeed, subtraction of a signal reproducing the electron energy-loss band from the thresholdphotoelectron spectrum, replicates qualitatively the $\mathrm{He}(\mathrm{I})$-photo-electron band shape. Nevertheless, the position in energy of all the features observed in the energy range of $14.7-17 \mathrm{eV}$ (marked by vertical lines in fig. 3 ) is in very good agreement with those measured in the He(I)-photoelectron spectrum. In fig. 3, the onset energies for $\mathrm{NH}_{2}{ }^{+}$and $\mathrm{NH}^{+}$fragment ions are inserted. From the foregoing discussion, autoionization would presumably contribute to the decay of $\mathrm{NH}_{3}{ }^{+}$in both channels.

The very weak band observed in the $22-29 \mathrm{eV}$ photon-energy range corresponds to the $\mathrm{NH}_{3}^{+}\left(\tilde{\mathrm{F}}^{2} \mathrm{~A}_{1}\right)$ ionic band observed in the He (II)-photoelectron spectrum of $\mathrm{NH}_{3}$ [ 12 ]. Compared to the latter spectrum, the 
band looks structureless also, but its intensity is weaker. Though it is difficult to estimate, this band spreads from about 23-30 eV. About the same range is observed in the He(II)-photoelectron spectrum. Very likely, only direct ionization phenomena are involved in this energy range.

\section{Acknowledgement}

We acknowledge the Bundesministerium für Forschung und Technologie and the Fonds National de la Recherche Scientifique for financial support. We are indebted to Dr. H.W. Jochims for his valuable assistance and helpful discussions. One of us (RL) wishes to thank the European Community for a grant (No. GE1-0018$\mathrm{D}(\mathrm{B}))$ in the framework of its LSI-program.

\section{References}

[1]] R. Locht, Ch. Servais, M. Ligot, Fr. Derwa and J. Momigny, Chem. Phys. 123 (1988) 443.

[2] R. Locht, Ch. Servais, M. Ligot, M. Davister and J. Momigny, Chem. Phys. 125 (1988) 425.

[3] R. Locht and J. Momigny, Chem. Phys. 127 (1988) 435.

[4] R. Locht, G. Hagenow, W. Denzer and H. Baumgärtel, Chem. Phys, to be submitted for publication.

[5] R. Locht, B. Leyh, W. Denzer, G. Hagenow and H. Baumgärtel, Chem. Phys. 155 (1991) 407.

[6] G. Hagenow, K. Hottmann and H. Baumgärtel, Chem. Phys. Letters 164 (1989) 395.

[7] K. Hottmann and H. Baumgartel, to be published.

[8] J.W. Rabalais, L. Karlsson, L.O. Werme, T. Bergmark and K. Siegbahn, J. Chem. Phys. 58 (1973) 3370.

[9] T. Baer, P.M. Guyon, I. Nenner, A. Tabche-Fouhaille, R. Botter, L.F.A. Ferreira and T.R.Govers, J. Chem. Phys. 70 (1979) 1585.

[10] D.M.P. Holland, J.B. West, A.C. Parr, D.L. Ederer, R. Stockbauer, R.D. Buff and J.L. Dehmer, J. Chem. Phys. 78 (1983) 124.

[11] M. Furlan, Thèse de doctorat, Université de Liège (1988).

[12] A.W. Potts, T.A. Williams and W.C. Price, Faraday Discussions Chem. Soc. 54 (1972) 104. 